Discrete Comput Geom 28:467-473 (2002)

DOI: $10.1007 / \mathrm{s} 00454-002-2882-5$

\title{
The Unit Distance Problem for Centrally Symmetric Convex Polygons
}

\author{
Bernardo M. Ábrego and Silvia Fernández-Merchant \\ Department of Mathematics, California State University, Northridge, \\ Northridge, CA 91330-8313, USA \\ \{bernardo.abrego,silvia.fernandez\}@csun.edu
}

\begin{abstract}
Let $f(n)$ be the maximum number of unit distances determined by the vertices of a convex $n$-gon. Erdős and Moser conjectured that this function is linear. Supporting this conjecture we prove that $f^{\mathrm{sym}}(n) \sim 2 n$ where $f^{\mathrm{sym}}(n)$ is the restriction of $f(n)$ to centrally symmetric convex $n$-gons. We also present two applications of this result. Given a strictly convex domain $K$ with smooth boundary, if $f_{K}(n)$ denotes the maximum number of unit segments spanned by $n$ points in the boundary of $K$, then $f_{K}(n)=O(n)$ whenever $K$ is centrally symmetric or has width $>1$.
\end{abstract}

\section{Introduction}

For every finite set of points $P$ in the plane, $f(P)$ denotes the number of unit segments with endpoints in $P$. We say that $P$ is in convex position if $P$ is the vertex set of a strictly convex polygon (no three points are on a line).

More than 40 years ago, Erdôs and Moser ([7], see also [8], [5], and [10]) initiated the study of the function

$$
f(n)=\max \{f(P):|P|=n, P \text { in convex position }\} .
$$

They proved with a construction that $f(n) \geq\lfloor 5 / 3(n-1)\rfloor$ and conjectured that $f(n)$ was linearly bounded above. The best known upper bound, $f(n) \leq O(n \log n)$, was first proved by Füredi [9], and very recently by Braß et al. [2] and Braß and Pach [3] using different techniques. The lower bound was improved to $2 n-7$ by Edelsbrunner and Hajnal [4], and, motivated by this construction, Erdôs and Fishburn [6] conjectured that $f(n)<2 n$.

Our main objective is to prove that $f(n)$ restricted to centrally symmetric sets is asymptotically $2 n$. This supports both the Erdôs-Moser and the Erdôs-Fishburn 
conjectures. In fact, we prove that the function

$$
f^{\text {sym }}(n)=\max \{f(P):|P|=n, P \text { in convex position and centrally symmetric }\}
$$

(which only makes sense for even values of $n$ ) satisfies

Theorem 1. For every even $n \geq 2$,

$$
2 n-\Theta(\sqrt{n}) \leq f^{\mathrm{sym}}(n) \leq 2 n-3 .
$$

None of the two constructions mentioned above giving lower bounds for $f(n)$ can be extended to a centrally symmetric set. Actually, the natural example consisting of the symmetrization of rotated copies of a regular triangle sharing a vertex, only gives $\frac{3}{2} n \leq f^{\text {sym }}(n)$. Even with this in mind, and due in part to the proof of Theorem 1, we conjecture that $f^{\text {sym }}(n) \geq 2 n-O(1)$.

The proof of the upper bound in Theorem 1 can be extended to a more general result stated below as Theorem 2 . We first need to define a family of functions. Let $K$ denote a strictly convex domain in the plane (i.e., a bounded subset of the plane such that if $x$ and $y$ are boundary points of $K$, then the open segment $x y$ is contained in the interior of $K$ ) with smooth boundary $\partial K$. Define

$$
f_{K}(n)=\max \{f(P):|P|=n \text { and } P \subset \partial K\},
$$

i.e., $f_{K}(n)$ is the maximum number of unit distances determined by $n$ boundary points of $K$.

Theorem 2. If $K$ is centrally symmetric, then $f_{K}(n) \leq 2 n-3$ for every $n \geq 2$.

We go one step further in this direction by considering a different family of sets $K$. The following is also an application of Theorem 1.

Theorem 3. If $K$ has width greater than one, then there is $c>0$ such that $f_{K}(n) \leq c n$.

It may be possible to prove the Erdôs-Moser conjecture by showing $f_{K}(n) \leq c n$ for a large class of convex sets $K$ and a universal constant $c$. Unfortunately, for these purposes, in Theorem $3 c \rightarrow \infty$ when the width approaches one.

From now on given any points $x, y$ in the plane, $H^{+}(x, y)$ and $H^{-}(x, y)$ denote the upper and lower half-planes determined by the oriented line $\overrightarrow{x y}$ (we include the line $x y$ in both half-planes).

\section{Proofs of the Theorems}

Proof of Theorem 1. We first prove the upper bound. Let $P$ denote a convex centrally symmetric polygon with $n$ vertices, and let $p q$ be a diameter of $P$. Note that if $\|p-q\|<$ 1 , then $f(P)=0$. We claim that

$$
\text { if }\|p-q\| \geq 1, \quad \text { then } f(P) \leq 4+f(P \backslash\{p, q\})
$$

which by induction implies $f(P) \leq 4+2(n-2)-3=2 n-3$.

To verify (1) it is enough to show that at most one point in $P \cap H^{+}(p, q)$ is at 
distance one from $p$. Assume that the origin $o$ is the center of symmetry of $P$. Observe that $q=-p$, otherwise one of the diagonals of the parallelogram $p q(-p)(-q)$ would be longer than the diameter $p q$. Moreover, since $P$ is centrally symmetric, $P$ must be contained in the closed disk $D$ determined by the circle through $p$ and $q$ centered at $o$. Let $C$ be the unit circle with center at $p$, and $u=C \cap p q$. Note that if $p_{1}, p_{2} \in$ $P \cap C \cap H^{+}(p, q)$ and $\left\|p_{1}-u\right\|>\left\|p_{2}-u\right\|$, then $\measuredangle p p_{1} p_{2}<\pi / 2$ since $\triangle p p_{1} p_{2}$ is isosceles, but $\measuredangle p p_{1} q \geq \pi / 2$ since $p_{1} \in D$. Therefore $p_{2}$ would be in the interior of $\triangle p q p_{1}$ contradicting the convexity of $P$ (when $\|p-q\|=1$ the only possibility is $\left.p_{1}=p_{2}=q\right)$.

To prove the lower bound we construct a centrally symmetric convex polygon $P$ with $n=k^{2}+k$ vertices and at least $2 n-3 k$ unit distances among them.

We start with $k$ points in a circle of radius $\frac{1}{2}$. Even though we look at these points as vectors, for simplicity we write their polar coordinates to describe them. Given a fixed $\theta \in(0, \pi)$ let

$$
p_{j}=\left(\frac{1}{2}, \theta_{j}\right), \quad \text { where } \theta_{1}=0 \text { and } \theta_{j}=7^{j-k} \theta \text { for } \quad 2 \leq j \leq k .
$$

Now, for every pair $(i, j)$ with $1 \leq i<j \leq k$, there is a unique point $p_{i, j}$ in $H^{+}\left(-p_{1}, p_{1}\right)$ obtained as the intersection of the unit circles with centers $-p_{i}$ and $-p_{j}$ (see Fig. 1(a)). Suppose that $\left(r_{i, j}, \theta_{i, j}\right)$ are the polar coordinates of $p_{i, j}$. By construction we have that $\theta_{i, j}=\left(\theta_{i}+\theta_{j}\right) / 2$, and after some direct calculations

$$
r_{i, j}=\frac{1}{2}\left(\sqrt{3+\cos ^{2}\left(\left(\theta_{j}-\theta_{i}\right) / 2\right)}-\cos \left(\left(\theta_{j}-\theta_{i}\right) / 2\right)\right) .
$$

Let $P=\bigcup_{1 \leq i<j \leq k}\left\{p_{i, j},-p_{i, j}\right\} \cup \bigcup_{1 \leq i \leq k}\left\{p_{i},-p_{i}\right\}$. Clearly, $P$ is centrally symmetric and $|P|=2\left(\left(\begin{array}{c}k \\ 2\end{array}\right)+k\right)=n$. Also, each point $p_{i, j}$ is at distance one from $-p_{i}$ and $-p_{j}$ which together with the symmetric analogues gives $4\left(\begin{array}{l}k \\ 2\end{array}\right)$ unit distances. If we add the
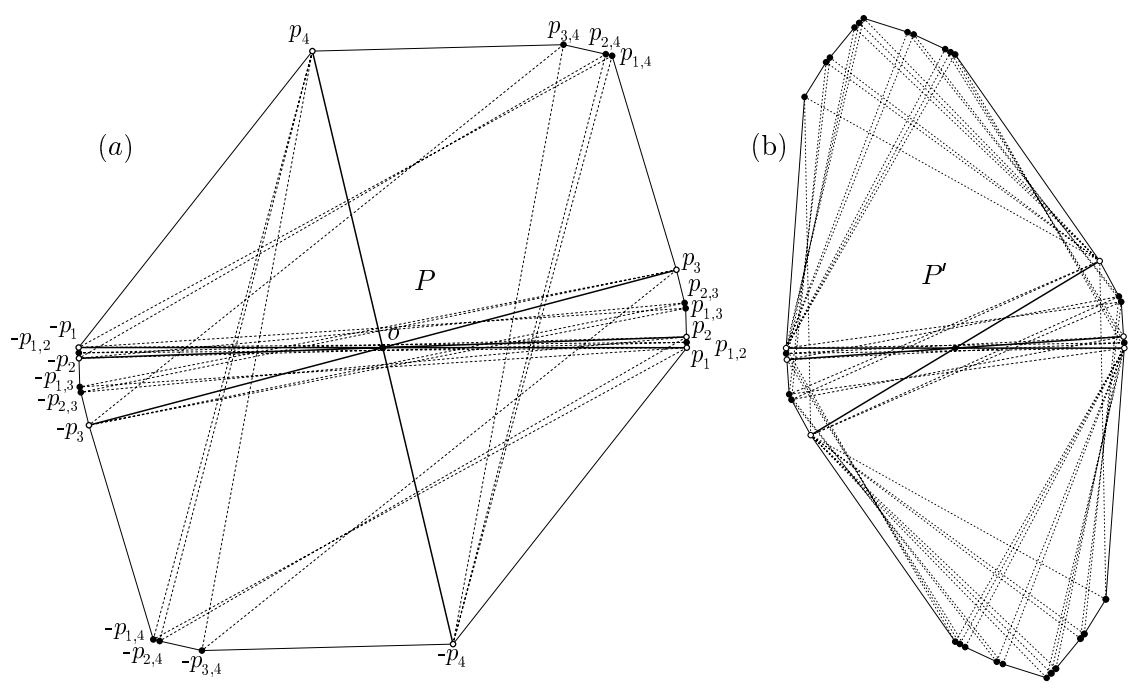

Fig. 1. Constructions for the lower bound of Theorem 1. 
$k$ unit distances given by the pairs $\left(p_{i},-p_{i}\right)$, we get $f(P) \geq 4\left(\begin{array}{l}k \\ 2\end{array}\right)+k=2 n-3 k \geq$ $2 n-3 \sqrt{n}$. Finally, we argue that $P$ is in convex position.

According to the angles $\theta_{i, j}$ we know that the points

$$
p_{1}, p_{1,2}, p_{2}, p_{1,3}, p_{2,3}, p_{3}, \ldots, p_{k-1}, p_{1, k}, p_{2, k}, p_{3, k}, \ldots, p_{k-1, k}, p_{k},-p_{1}
$$

are in $H^{+}\left(-p_{1}, p_{1}\right)$, and they appear in this order. So by symmetry we just need to show that these $n / 2+1$ points are in convex position and $\measuredangle p_{1,2} p_{1} o, \measuredangle o\left(-p_{1}\right) p_{k}<\pi / 2$. Observe that the points $p_{1, j}, p_{2, j}, \ldots, p_{j-1, j}, p_{j}$ are contained in an arc of circle with center at $-p_{j}$, and thus they are in convex position. Also $\measuredangle o\left(-p_{1}\right) p_{k}=\measuredangle\left(-p_{1}\right) p_{k} o<$ $\pi / 2$ and for all $2 \leq j \leq k, \measuredangle o p_{j} p_{j-1, j}=\measuredangle\left(-p_{j}\right) p_{j} p_{j-1, j}<\pi / 2, \measuredangle p_{2, j} p_{1, j} o<$ $\measuredangle p_{2, j} p_{1, j}\left(-p_{j}\right)<\pi / 2$ (here $p_{2,2}=p_{2}$ ).

So it is enough to prove that $\measuredangle o p_{1, j} p_{j-1}<\measuredangle p_{1, j} p_{j-1} o<\pi / 2$ for $2 \leq j \leq k$. The first inequality is given by $\left\|p_{1, j}\right\|>\left\|p_{j-1}\right\|$, and the second is equivalent to showing that $\left\langle-p_{j-1}, p_{1, j}-p_{j-1}\right\rangle>0$, where $\langle\ldots$,$\rangle denotes the standard inner product. When$ $j=2$ we have $\measuredangle p_{1,2} p_{1} o=\measuredangle p_{1,2} p_{1}\left(-p_{1}\right)<\pi / 2$, and for $j \geq 3$,

$$
\begin{aligned}
\left\langle-p_{j-1}, p_{1, j}-p_{j-1}\right\rangle & =\left\langle p_{j-1}, p_{j-1}\right\rangle-\left\langle p_{j-1}, p_{1, j}\right\rangle \\
& =\left\|p_{j-1}\right\|^{2}-\left\|p_{j-1}\right\|\left\|p_{1, j}\right\| \cos \left(\theta_{1, j}-\theta_{j-1}\right) \\
& =\frac{1}{4}-\frac{1}{2} r_{1, j} \cos \left(\theta_{j} / 2-\theta_{j-1}\right) \\
& =\frac{1}{4}\left(1-\cos \left(\theta_{j} / 2-\theta_{j-1}\right)\left(\sqrt{3+\cos ^{2}\left(\theta_{j} / 2\right)}-\cos \left(\theta_{j} / 2\right)\right)\right),
\end{aligned}
$$

by construction $\theta_{j}=7 \theta_{j-1}$, thus

$$
\left\langle-p_{j-1}, p_{1, j}-p_{j-1}\right\rangle=\frac{1}{4}\left(1-\cos \left(\frac{5}{2} \theta_{j-1}\right)\left(\sqrt{3+\cos ^{2}\left(\frac{7}{2} \theta_{j-1}\right)}-\cos \left(\frac{7}{2} \theta_{j-1}\right)\right)\right) .
$$

To complete the proof note that the function $g(x)=1-\cos (2.5 x)\left(\sqrt{3+\cos ^{2}(3.5 x)}-\right.$ $\cos (3.5 x))$ is positive in the interval $(0, \pi / 7)$ and $\theta_{j-1} \leq \theta_{k-1}=\theta / 7<\pi / 7$.

Remark. We can reduce the error for the lower bound by adding some points to our original construction (see Fig. 1(b)). Given $p \in P$ let $C(p)$ be the unit circle centered at $p$ and

$$
h(p)= \begin{cases}C(p) \cap C\left(-p_{1}\right) \cap H^{+}\left(-p_{1}, p_{1}\right) & \text { if } p \in H^{+}\left(-p_{1}, p_{1}\right) \backslash\left\{-p_{1}\right\}, \\ C(p) \cap C\left(p_{k}\right) \cap H^{+}\left(-p_{1}, p_{1}\right) & \text { otherwise. }\end{cases}
$$

Let $P^{\prime}=P \cup \bigcup_{p \in P}\{-h(p), h(p)\}$. For $\theta$ small enough, it can be verified that $P^{\prime}$ is in convex position. Also $\left|P^{\prime}\right|=3|P|-2=3 k^{2}+3 k-2=n^{\prime}$ and $f\left(P^{\prime}\right) \geq$ $f(P)+2(2|P|-2) \geq 2 n^{\prime}-3 k \geq 2 n^{\prime}-\sqrt{3 n^{\prime}}$.

Finally, by deleting an appropriate number of points from $P^{\prime}$, one can show that for an arbitrary even $n \geq 2, f^{\text {sym }}(n) \geq 2 n-3-\sqrt{3 n}$.

Proof of Theorem 2. Consider any $n$-point subset $P$ of $\partial K$. Let $P^{\prime}$ be the symmetric of $P$ in $\partial K$. The previous proof guarantees that each of the endpoints of the diameter of $P \cup P^{\prime}$ is at distance one of at most two other elements in $P \cup P^{\prime}$. Moreover, one of these points is in $P$. The rest follows by induction. 

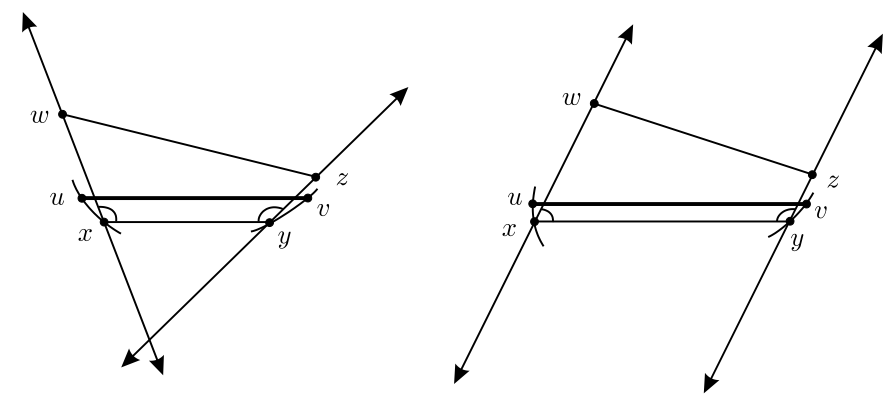

Fig. 2. The chord $u v$ is larger than the chord $x y$.

Proof of Theorem 3. The directed closed segment $x y$ is a chord of $K$ in direction $\alpha$ if $x, y \in \partial K$ and the argument of the vector $y-x$ is $\alpha$. For each $\alpha \in[0,2 \pi)$ we say that $x y$ is the $\alpha$-directional diameter of $K$, or simply the $\alpha$-diameter, if $x y$ is the longest chord of $K$ in direction $\alpha$ (this is well defined because $K$ is strictly convex). We also denote by $a_{\alpha}, b_{\alpha}$ the endpoints of the unique unit chord of $K$ in direction $\alpha$ with the property that any chord parallel to $a_{\alpha} b_{\alpha}$ contained in $H^{-}\left(a_{\alpha}, b_{\alpha}\right)$ has length less than one. We call $a_{\alpha} b_{\alpha}$ the $\alpha$-unit chord of $K$. We need the next lemma for the proof of the theorem.

Lemma 1. Any two directional diameters of $K$ intersect in their interior.

Proof. Let $x y$ and $z w$ be the $\alpha$ - and $\beta$-diameters of $K$ and suppose that they not intersect. Then the quadrilateral with vertices $x, y, z, w$ is convex and $x y, z w$ are opposite sides (see Fig. 2). Assume that $x y z w$ is the order of the vertices in the quadrilateral. Since the internal angles add up to $2 \pi$ then we can assume that $\measuredangle y x w+\measuredangle z y x \geq \pi$. Since $K$ is strictly convex then there is a chord parallel to $x y$ in $H^{+}(x, y)$ with length greater than $x y$ which contradicts the fact that $x y$ is the $\alpha$-diameter. Finally note that even if $x=w$ (or $z=y$ ), we can replace the line $x w$ (or $z y$ ) by the tangent line to $K$ at $x$ (or at $y$ ) and the argument still follows (here we use the smoothness assumption).

The last lemma, together with the continuity of $\partial K$, guarantees that for any boundary point $x$ of $K$ there exists a unique directional diameter with $x$ as one of its endpoints. It also shows that both the left and right endpoints of the $\alpha$-diameter (as functions of $\alpha$ ) move continuously counterclockwise in $\partial K$. For each $\alpha$-unit chord look at the two directional diameters having $a_{\alpha}$ or $b_{\alpha}$ as one of their endpoints, and let $c_{\alpha}$ be their point of intersection. Let $\theta(\alpha)=\pi-\angle b_{\alpha} c_{\alpha} a_{\alpha}$ (see Fig. 3). Since $K$ has width greater than one then $\theta$ is a strictly positive function. Hence, by continuity of $\theta$ on the compact set $[0,2 \pi]$ we have $m=\min \{\theta(\alpha): \alpha \in[0,2 \pi]\}>0$.

Let $P$ be an $n$-point subset of $\partial K$. Define

$$
U=\left\{\alpha \in[0,2 \pi): a_{\alpha}, b_{\alpha} \in P\right\}
$$

and for every $\beta \in[0, \pi)$,

$N(\beta)=\mid\{\alpha \in U:$ the $\alpha$-unit chord does not cross the $\beta$-diameter $\} \mid$. 


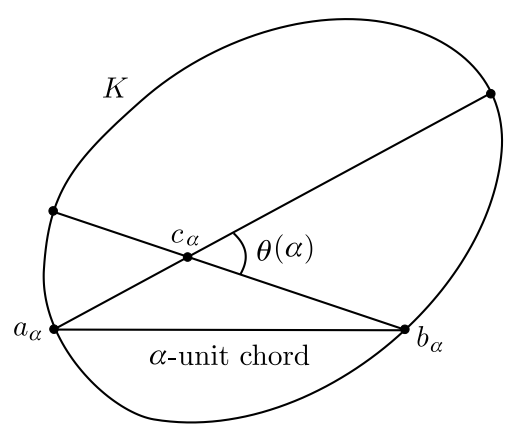

Fig. 3. Definition of $\theta(\alpha)$.

Observe that

$$
N(\beta)=\sum_{\alpha \in U} \chi_{\beta}(\alpha)
$$

where

$$
\chi_{\beta}(\alpha)= \begin{cases}1 & \text { if the } \alpha \text {-unit chord does not intersect the } \beta \text {-diameter } \\ 0 & \text { otherwise. }\end{cases}
$$

So

$$
\int_{0}^{\pi} N(\beta) d \beta=\int_{0}^{\pi} \sum_{\alpha \in U} \chi_{\beta}(\alpha) d \beta=\sum_{\alpha \in U} \int_{0}^{\pi} \chi_{\beta}(\alpha) d \beta,
$$

but for fixed $\alpha$, if $b_{\alpha}$ is an endpoint of the $\delta$-diameter, then $\chi_{\beta}(\alpha)=1$ if and only if $\beta \in[\delta, \delta+\theta(\alpha)]$, i.e., $\int_{0}^{\pi} \chi_{\beta}(\alpha) d \beta=\theta(\alpha)$. Therefore

$$
m f(P)=m|U| \leq \sum_{\alpha \in U} \theta(\alpha)=\int_{0}^{\pi} N(\beta) d \beta .
$$

Now, as an application of Theorem 1 we claim that

$$
N(\beta)<2 n \quad \text { for all } \beta \in[0, \pi)
$$

and so

$$
\int_{0}^{\pi} N(\beta) d \beta \leq 2 \pi n
$$

Thus $f(P) \leq(2 \pi / m) n$ for all $n$-point subsets of $\partial K$. Hence $f_{K}(n) \leq(2 \pi / m) n$.

To prove (2) let $x y$ be the $\beta$-diameter. First suppose that $|\{x, y\} \cap P| \leq 1$, let $P_{1}=$ $P \cap H^{+}(x, y), P_{2}=P \cap H^{-}(x, y)$, and $P_{1}^{\prime}, P_{2}^{\prime}$ be the sets obtained from $P_{1}$ and $P_{2}$ by symmetrization with respect to the midpoint of $x y$. Since $x y$ is a directional diameter then the sets $P_{1} \cup P_{1}^{\prime}$ and $P_{2} \cup P_{2}^{\prime}$ are in convex position, so according to Theorem 1, for $i=1,2$,

$$
2 f\left(P_{i}\right)=f\left(P_{i}\right)+f\left(P_{i}^{\prime}\right) \leq f\left(P_{i} \cup P_{i}^{\prime}\right) \leq 2\left|P_{i} \cup P_{i}^{\prime}\right|-3=4\left|P_{i}\right|-3,
$$


therefore

$$
N(\beta)=f\left(P_{1}\right)+f\left(P_{2}\right) \leq 2\left(\left|P_{1}\right|+\left|P_{2}\right|\right)-3 \leq 2 n-1<2 n .
$$

If $x, y \in P$, then $\left|P_{i} \cup P_{i}^{\prime}\right|=2\left|P_{i}\right|-2$ in the above analysis, so even though $\left|P_{1}\right|+\left|P_{2}\right|=$ $n+2$ the conclusion still holds.

Corollary 1. Let $K$ be a strictly convex domain with $C^{2}$ boundary. If the curvature of $K$ is less than two at each point of $\partial K$, then $f_{K}(n) \leq c n$ for some positive constant $c$ that only depends on $K$.

Proof. By Blaschke's Rolling Theorem [1] if the curvature of $K$ is less than two at each point of $\partial K$, then a circle of radius $\frac{1}{2}$ can freely roll inside $K$, and therefore the width of $K$ is greater than one.

\section{Acknowledgments}

We are grateful to Farhad Shahrokhi and László Székely for their efforts in organizing the material of this volume and to the referees for their valuable comments.

\section{References}

1. W. Blaschke (1956), Kreis und Kugel, 2. Auflage, Berlin: Walter de Gruyter.

2. P. Braß, Gy. Károlyi, and P. Valtr (2001), An extremal theory of convex geometric graphs and related structures, manuscript.

3. P. Braß and J. Pach (2001), The maximum number of times the same distance can occur among the vertices of a convex $n$-gon is $O(n \log n)$, Journal of Combinatorial Theory, Series A, 94, 178-179.

4. H. Edelsbrunner and P. Hajnal (1991), A lower bound on the number of unit distances between the points of a convex polygon, Journal of Combinatorial Theory, Series A, 56, 312-316.

5. P. Erdôs (1980), Some combinatorial problems in geometry, in Geometry and Differential Geometry, Lecture Notes in Mathematics, Vol. 792, Springer-Verlag, New York, pp. 46-53.

6. P. Erdôs and P. C. Fishburn (1995), Multiplicities of interpoint distances in finite planar sets, Discrete Applied Mathematics, 60, 141-147.

7. P. Erdős and L. Moser (1959), Problem 11, Canadian Mathematical Bulletin, 2, 43.

8. P. Erdős and L. Moser (1970), An extremal problem in graph theory, Australian Journal of Mathematics, 11, 42-47.

9. Z. Füredi (1990), The maximum number of unit distances in a convex $n$-gon, Journal of Combinatorial Theory, Series A, 55, 316-320.

10. J. Pach and P. K. Agarwal (1995), Combinatorial Geometry, Wiley-Interscience, New York.

Received June 1, 2001, and in revised form December 31, 2001. Online publication October 29, 2002. 